\title{
Study of the Estimation of Sound Source Signal Direction Based on MUSIC Algorithm
}

\author{
Bao-Hai YANG ${ }^{1, a,{ }^{*}, \text { Ze-Liang LIU }}{ }^{1, b}$ and Dong CHEN ${ }^{1, c}$ \\ ${ }^{1}$ College of Electronic Engineering, Jiujiang Univercity,Jiujiang,332005,China

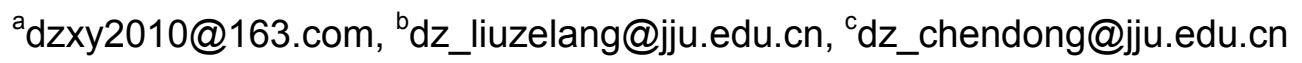

Keywords: Equally space dlinear array, MUSIC algorithm, Sound source signal, Direction estimation.

\begin{abstract}
The multiple signal classification, MUSIC algorithm, introduces linear space to direction estimation, which realizes the breakthrough of the sound source direction resolution and lays an important foundation for the method and theory of spacial spectrum estimation. In order to better use the algorithm to estimate the direction of the sound source signal, this paper illustrates equally spaced linear array based on the MUSIC algorithm model and carries out the comparative simulation study on the narrow-band and the broad-band sound source signal direction estimation. Computer simulation shows that MUSIC algorithm can achieve super resolution direction estimation of the narrowband signals, and when MUSIC algorithm is used to estimate the direction of broadband signals, its performance is greatly degraded. And finally the conclusion is that in the narrowband sound source signal direction estimation, MUSIC algorithm functions remarkably, and when handling voice and other broadband source signal direction estimation, it needs to be further improved.
\end{abstract}

\section{Introduction}

Sound source direction estimation is one of the hot areas in signal processing study in recent decades. It first collects voice signals, then uses digital signal processing technology to analyze and process them and achieves the signal spatial spectrum, and finally estimates the sound source direction. The sound source direction estimation mainly researches super-resolution estimation, multiple signal classification (MUSIC) algorithm proposed by Schmidt and others -- one of the algorithms to achieve super-resolution direction estimation[1-3]. MUSIC algorithm relates to not only the direction vector and array structure, but the signal frequency as well [4-5].

\section{Characteristics and principles of MUSIC algorithm}

MUSIC algorithm is characterized by high spatial spectrum resolution and asymptotical unbiased estimation for the number of sources, DOA, background noise level and signal strength. It can achieve multi-channel DOA estimation. If signal subspace is smaller than noise subspace, that is the source number is less than half of array elements, MUSIC algorithm has better estimation performance[6,7].

The basic principle of MUSIC algorithm is as follows. When signal and background noise satisfy certain conditions, and the source number is less than that of the array elements, eigenvalue decomposition can be used to decompose the correlation matrix data output from the matrix elements and they are decomposed into signal subspace and noise subspace, which are orthogonal[8]. When the signal to noise ratio is sufficient, the signal subspace's eigenvalue will be larger than the noise subspace eigenvalue, thus it is easy to distinguish the signal subspace and noise subspace, the subspace rank is less than that of the autocorrelation matrix. Using signal or noise subspace and the vector direction to form a spatial spectrum, and scan, when scanning DOA, the spacial spectrum will achieve larger value and the signal direction estimation will be acquired[9]. 


\section{MUSIC algorithm}

Currently, the theory and method of using array to estimate the direction has layed theoretical foundation for estimation of the sound source signal direction. MUSIC algorithm based on traditional model of equally spaced linear array (Figure 1) introduces the concept of the linear space into direction estimation. This will realize the breakthrough of the resolution in sound source direction.

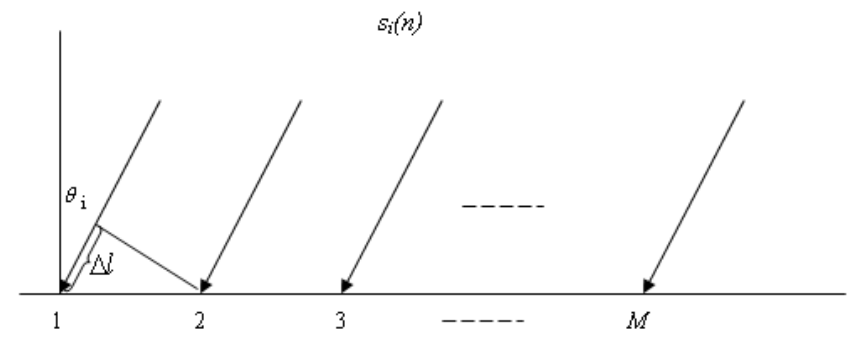

Fig.1 Traditional model of equally spaced linear array

One sample is called one snapshot. Suppose N snapshots of the received signals were observed in each array element. Using these observed values to construct correlation matrix, the desired signal $\mathrm{s}(\mathrm{n})$ 's DOA -- $\theta$-- can be achieved.Suppose

$$
R_{X X}=\frac{1}{N} \sum_{n=1}^{N} x(n) x^{H}(n)
$$

Equation (1) is the sample autocorrelation matrix of the observed signal vector. The ideal autocorrelation matrix is impossible to get, so more snapshots should be used to estimate the autocorrelation matrix, that is the sample autocorrelation matrix. When the snapshot number tends to infinity, the sample autocorrelation matrix will converge to an ideal auto-correlation matrix with the probability 1 , because the signal is stationary,For the array signal observation model described by equation $x(n)=\sum_{i=1}^{p} \alpha\left(\omega_{i}\right) s_{i}(n)+e(n)=A(\omega)+e(n)$, the following assumptions can be made.

The equally spaced linear array, for different $\omega_{\mathrm{i}}$, vectors $\alpha\left(\omega_{\mathrm{i}}\right)$ are unrelated. That is the direction matrix is a full column rank matrix. Each element of additive noise vector is zero mean of Gaussian white noise, uncorrelated, and has the same variance, satisfying (2); In which $\sigma^{2}$ is the additive noise variance.

$$
E\{e(n)\}=0, E\left\{e(n) e^{H} e(n)\right\}=\sigma^{2} I
$$

Singal sources are independent of each other, that is to say the signal source derived from the correlation matrix is the non-singular matrix, but full matrix, satisfying (3); The signal (3)source and noise are independent of each other and satisfy equation (4). Under the assumed conditions, from equation (1), the following (5) is achieved.

$$
\begin{aligned}
& \operatorname{rank}\left(s(n) s^{H}(n)\right)=p \\
& \left.E\left\{s(n) e^{H} n\right)\right\}=0, E\left\{e(n) s^{H}(n)\right\}=0 \\
& \left.R x x=E\left\{x(n) x^{H} n\right)\right\}=E\left\{[A(\omega) s(n)+e(n)][A(\omega) s(n)+e(n)]^{H}\right\}=A P A^{H}+\sigma^{2} I
\end{aligned}
$$

Among which, $A=A(\omega) P=E\left\{s(n) S^{H}(n)\right\}$. So $R x x$ is the symmetric matrix of a full matrix. When decompose its eigenvalue, the following equation can be acquired.

$$
R x x=U \Sigma U^{H}, \Sigma=\operatorname{diag}\left(\sigma_{1}{ }^{2}, \cdots, \sigma_{M}^{2}\right)
$$

The total number of $R x x$ 's eigenvalues is M. (5) multiplies $U^{H}$ left and $U$ right, equation (7) can be acquired, (6) multiplies $U^{H}$ left and $U$ right, equation (8) can be acquired.

$$
\begin{aligned}
& U^{H} R x x U=U^{H}\left(A P A^{H}+\sigma^{2} I\right) U \\
& U^{H} R x x U=U^{H} U \sum U^{H} U=\Sigma
\end{aligned}
$$

With equations (7) and (8), (9) can be acquired. The vector matrix is a full matrix, so $\sum=U^{H} A P A^{H} U+U^{H} \sigma^{2} I U=U^{H} E\left\{A s(n) s^{H}(n) A^{H}\right\} U+U^{H} \sigma^{2} I U=\operatorname{diag}\left(\sigma_{1}{ }^{2}, \cdots \sigma M^{2}\right)$ 


$$
\boldsymbol{U}^{\mathrm{H}} \boldsymbol{A} \boldsymbol{P} \boldsymbol{A}^{\mathrm{H}} \boldsymbol{U}=\boldsymbol{U}^{\mathrm{H}} E\left\{\boldsymbol{A s}(n) \boldsymbol{s}^{\mathrm{H}}(n) \boldsymbol{A}^{\mathrm{H}}\right\} \boldsymbol{U}=\operatorname{diag}\left(a_{1}^{2}, \cdots a_{p}^{2}, 0, \cdots 0\right)
$$

In which $\alpha_{1}{ }^{2}, \cdots \alpha_{\mathrm{P}}{ }^{2}$ are autocorrelation matrix $A P A^{H}$, s eigenvalues of the observed signals $A s(n)$, when there is no additive noise. So with equations (9) and (10), the eigenvalues of the observed signals $x(n)$ 's autocorrelation matrix will be achieved.

$$
\lambda_{i}=\sigma_{i}=\left\{\begin{array}{l}
\alpha i^{2}+\sigma^{2}, i=1, \cdots p \\
\sigma^{2}, i=p+1, \cdots M
\end{array}\right.
$$

Equation (11) shows that, when there is additive observed white noise in the background, the autocorrelation matrix's eigenvalues of the observed vectors $x(n)$ are composed by two parts. The former p's eigenvalues are the total values of $\alpha_{\mathrm{i}}{ }^{2}$ and $\sigma^{2}--$ the variance of the additive white noise, and the latter M-p's are the variance of the additive white noise's variance $\sigma^{2}$.

When the signal to the noise ratio is sufficient, $\alpha_{\mathrm{i}}{ }^{2}>\sigma^{2}$, it is easy to distinguish $R x x$ 's larger $\mathrm{p}$ eigenvalues $\alpha_{\mathrm{i}}{ }^{2}+\sigma^{2}$ and M-P smaller ones $\sigma^{2}$. These main eigenvalues $\mathrm{P}$ are the signal eignevalues, and $\mathrm{M}-\mathrm{p}$ are the noise eigenvalues. With these signal eigenvalues and noise ones, characteristic matrix $U$ 's column vectors can be divided two parts. They are

$$
\begin{aligned}
& S=\left[s_{1}, \ldots s_{p}\right]=\left[u_{1}, \ldots u_{p}\right] \\
& G=\left[g_{1, \cdots} g_{M}-p\right]=\left[u_{p+1}, \cdots u_{M}\right] \\
& U=[S G]
\end{aligned}
$$

In which, $\mathrm{u}$ is U's column vector, $S$ is the signal eigenvector and $\mathrm{G}$ is the noise eigenvector. The space $\operatorname{span}\left\{s_{1}, \cdots, s_{p}\right\}$ composed by $S$ is called the signal subspace and $\operatorname{span}\left\{g_{1}, \cdots g_{M}-p\right\}$ by $G$ is noise subspace. With (6) , (15) can be achieved.

$$
U U^{H}=U^{H} U=I
$$

So $U$ is a unitary matrix, according the feathure of the unitary matrix, the signal subspace and

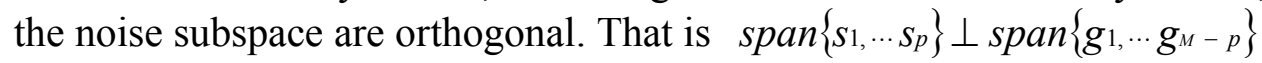

With equations (12), (13) and (15), equation (16) can be achieved.

$$
\operatorname{span}\left\{u_{1}, \cdots u_{p}\right\} \perp \operatorname{span}\left\{u_{p}+1, u_{M}\right\}
$$

If $G$ multiplies $R x x$ right, with equations (2.5), (2.6), (14) and (16), the following two equations can be acquired.Thus (18)

$$
\begin{aligned}
& R x x G=A P A{ }^{H} G+\sigma^{2} G A P A{ }^{H} G+\sigma^{2} G=[S G] \Sigma\left[\begin{array}{l}
S^{H} \\
G^{H}
\end{array}\right] G=[S G] \Sigma\left[\begin{array}{l}
0 \\
I
\end{array}\right]=\sigma^{2} G \\
& A P A{ }^{H} G=0, G^{H} A P A{ }^{H} G=0
\end{aligned}
$$

$P$ is full rank matrix, the determinant is not 0, so $A^{H} G=0$

When equation (11) is substituted into $A^{H} G=0,(19)$ is acquired.

$\alpha^{H}(\omega) G=0, \omega=\omega_{1} \ldots \omega_{p}$

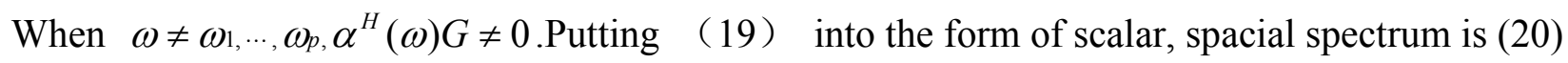

$P(\omega)=\frac{1}{\alpha^{H} G G^{H} \alpha(\omega)}$

From equation (20), the $\mathrm{p} \max \omega_{1}, \cdots, \omega_{p}$ can be achieved and the $\mathrm{p}$ DOA are $\theta_{1}, \cdots, \theta_{p}$. And finally, MUSIC algorithm is concluded as follows.

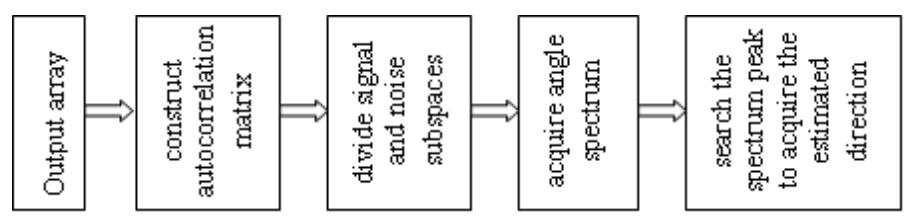

Fig.2 MUSIC algorithm processes 


\section{Computer simulation}

The simulation conditions are as follows. 8 array elements equally spaced linear array, angle searching ranges are $-90^{\circ} \sim 90^{\circ}$, searching step width is 1 ; incidence angle is $45^{\circ}$, the background noise is the Gaussian white noise, the signal to noise ratio is $20 \mathrm{~dB}$; signal center frequency is $1 \mathrm{KHz}$, space separation between array elements is half of the center frequency to wave length, that is $0.171 \mathrm{~m}$.

The modulating signal(21), The sound carrier(22),FM signal(23)

$$
\begin{aligned}
& u_{\Omega}(t)=2 \cos (2 \pi 10 t) \\
& u_{c}(t)=14 \cos \left(2 \pi 10^{3} t\right) \\
& u_{F M}(t)=14 \cos \left(2 \pi 10^{3} t+m_{f} \sin 2 \pi 10 t\right)
\end{aligned}
$$

In the equations $m_{f}$ is the FM index. When $m_{f}$ is far smaller than 1 , it is called narrowband FM signal; and when much larger than 1 , the broadband one.

(1) Estimation of narrowband signal direction

When $m_{f}=0.01$, the narrowband signal power spectrum is as fig 3 (Narrow band signal power spectrum), and the direction estimation simulation is as fig 4(Direction estimation using MUSICAlgorithm for narrow-band signal based on linear array)
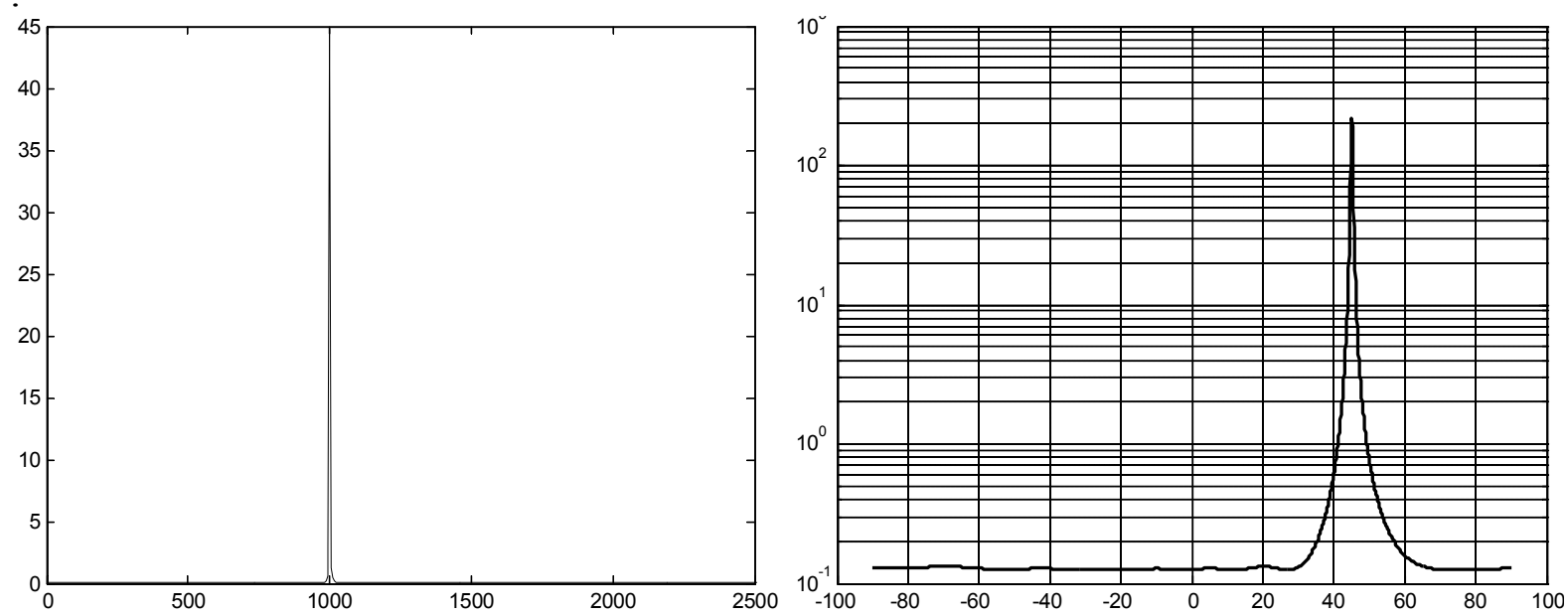

Fig.3 Narrow band signal power spectrum Fig.4 Direction estimation using MUSIC algorithm

(2) Estimation of broadband signal direction

When $m_{f}=100$, the broadband signal power spectrum is as Fig 5 (Wideband signal power spectrum), and the direction estimation simulation is as Fig 6(Direction estimation using MUSIC Algorithm for wideband signal based on linear array).
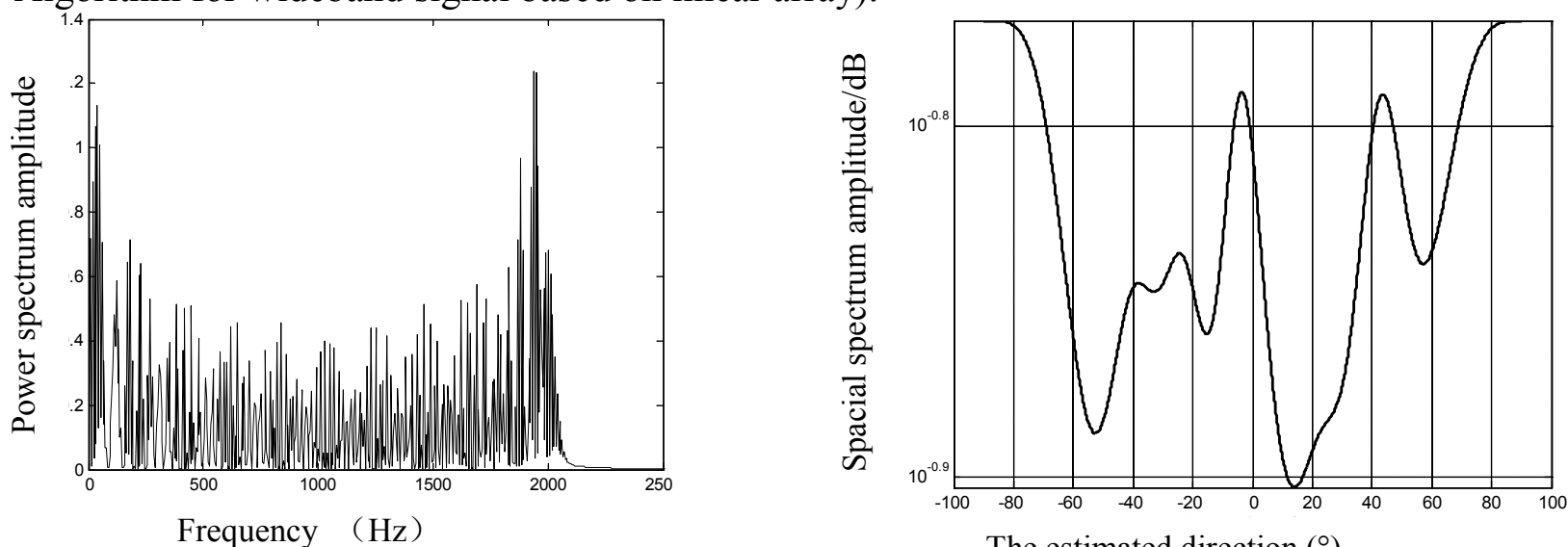

The estimated direction $\left(^{\circ}\right)$

Fig.5 Wideband signal power spectrum Fig. 6 Direction estimation using MUSIC Algorithm 
The causes for the incorrect estimation are as follows. The direction vector chooses the center frequency, while the broadband signal frequency covers broadly, so that the maximum signal frequency and the lowest number of frequency will greatly deviated from center frequency. For the high-frequency components, it results in the large array element spacing, which leads to false peaks. For the low-frequency components, it leads to much smaller array element spacing, which results in peak broadening. This results in MUSIC algorithm's significant performance degradation when estimating broadband signal direction.

\section{Conclusion}

Analysis shows that the MUSIC algorithm can achieve super-resolution signal direction estimation of the narrowband. If the signal bandwidth increases, MUSIC algorithm estimation performance will be greatly degraded. In practice, there are lots of broadband signals, such as estimation of the voice signal direction. It can improve MUSIC algorithm with the combination of the characters of the voice signal processing. In order to improve voice signal and other broadband signal direction estimation performance, the algorithm can be further studied and improved to adapt to source signal direction estimation of the broadband array.

\section{Acknowledgment}

This work is supported by Science Foundation of Jiangxi Provincial Department of Education (GJJ14739、GJJ14721), National Science Foundation of Jiangxi Province (20142BAB207006, 20151BAB207013),Science Foundation of Jiujiang Univercity (2013KJ02、2013KJ01).

\section{References}

[1]Carter C.The generalized correlation method for estimation of time delay[J].IEEE Transactions on Aeoust Peeeroeessn,1976,24(04):24--27.

[2]Gerhard D.Beamforming with Optimized Interpolated Microphone Arrays[J].Institute of Communications and Radio-Frequency Engineering,2008,1:33-36.

[3]Ju T L,Peng Q C,Shao H Z.Speech source localization sub-space algorithm research based on microphone array in near field[C].ICEMI NJTU,Beijing,2005:45-48.

[4]Ephraim Y,Van Trees H.A signal subspace approach for speech enhancement[J].IEEE Transactions on Speech and Audio Processing,1995,3(4):251-266.

[5]Rubsamen M,Gershman A B. Direction-of-arrivalestimation for nonuniform sensor arrays:from mani-fold separation to Fourier domain music methods[J] .IEEE Trans Signal Process, 2009, 57 (2) :588-599 .

[6]Unnikrishna S,Kwon B H.Forward/Backward Spatial Smoothing Techniques for Coherent Signal Identification[J].IEEE Transactions on ASSP,1989,37(1):8-15.

[7]Kundu D.Modified MUSIC Algorithm for Estimating DOA of Signals[J]. Signal Processing, 1996,48(1):85-90.

[8]Flanagan J L,Johnston J D,Zahn R,et al.Computer steered microphone arrays for sound transduction in large rooms[J].JAcoust Soc Amer,1985,78(5):1508-1518.

[9]Asano F,Asoh H,Matsui T.Sound source localization and separation in near field[J].IEEE

Transactions on Fundamentals of Electronics, 2000, E83-A(11):2286-2294. 